\title{
Sivers asymmetry for the proton and the neutron
}

\author{
S. Scopetta, , A. Courtoy , F. Fratini and V. Vento "\# \\ Dipartimento di Fisica, Università degli Studi di Perugia, via A. Pascoli, 06100 Perugia, Italy \\ ${ }^{\dagger}$ INFN, sezione di Perugia, via A. Pascoli, 06100 Perugia, Italy \\ Departament de Fisica Teòrica, Universitat de València \\ and Institut de Fisica Corpuscular, Consejo Superior de Investigaciones Científicas \\ 46100 Burjassot (València), Spain \\ ¥ TH-Division, PH Department, CERN, CH-1211 Genève 23, Switzerland
}

\begin{abstract}
.
A formalism is presented to evaluate the Sivers function in constituent quark models. A nonrelativistic reduction of the scheme is performed and applied to the Isgur-Karl model. The results obtained are consistent with a sizable Sivers effect and the signs for the $u$ and $d$ flavor contributions turn out to be opposite. The Burkardt Sum Rule is fulfilled to a large extent. After the estimate of the QCD evolution of the results from the momentum scale of the model to the experimental one, a reasonable agreement with the available data is obtained. A calculation of nuclear effects in the extraction of neutron single spin asymmetries in semi-inclusive deep inelastic scattering off ${ }^{3} \mathrm{He}$ is also described. In the kinematics of forth-coming experiments at JLab, it is found that the nuclear effects arising within an Impulse Approximation approach are under control.
\end{abstract}

Keywords: DIS, transversity, neutron structure

PACS: $13.88 .+\mathrm{e}, 12.39 .-\mathrm{x}, 21.45 .-\mathrm{v}$

\section{THE SIVERS FUNCTION IN CONSTITUENT QUARK MODELS}

The partonic structure of transversely polarized nucleons is one of their less known features (for a review, see, e.g., Ref. [1]). The work presented here aims to contribute to the effort of shedding some light on it.

Semi-inclusive deep inelastic scattering (SIDIS) is one of the proposed processes to access the parton distributions (PDs) of transversely polarized hadrons. SIDIS of unpolarized electrons off a transversely polarized target shows azimuthal asymmetries, the so called "single spin asymmetries" (SSAs) [2]. The SSAs are due to two physical mechanisms, whose contributions can be distinguished [3, 4, 5, 6]. One of them is the Collins mechanism, due to parton final state interactions (FSI) in the production of a hadron by a transversely polarized quark [2]. The other is the Sivers mechanism [7], producing a term in the SSA which is given by the product of the unpolarized fragmentation function with the Sivers PD, describing the number density of unpolarized quarks in a transversely polarized target. The Sivers function is a time-reversal odd, Transverse Momentum Dependent (TMD) PD; for this reason it was believed to vanish due to time reversal invariance. However, this argument was invalidated by a calculation in a spectator model [8], following the observation of the existence of leading-twist Final State Interactions (FSI) [9]. The current wisdom is that a non-vanishing Sivers function is generated by the gauge link in the definition of TMDs [10, 11, 12, 13], whose contribution does not vanish in the light-cone gauge, as happens for the standard 


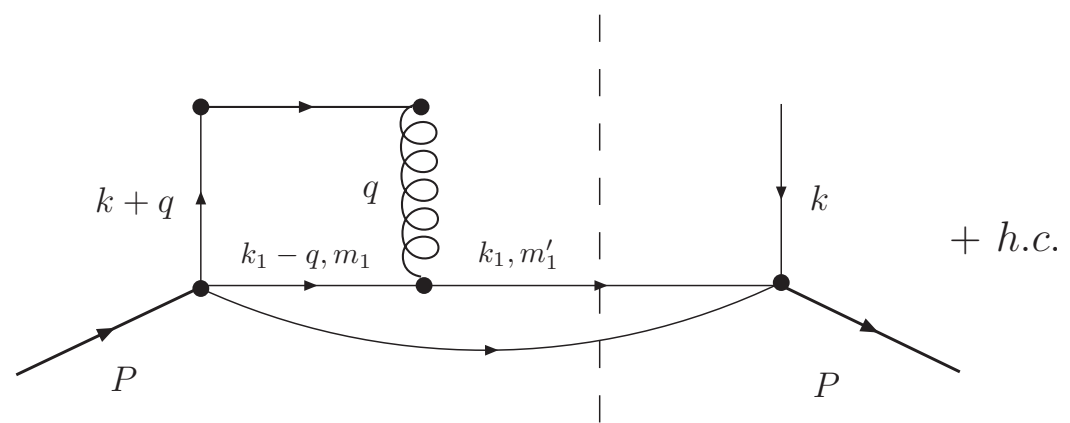

FIGURE 1. The contributions to the Sivers function in the present approach.

PD functions. Recently, the first data of SIDIS off transversely polarized targets have been published, for the proton [14] and the deuteron [15]. It has been found that, while the Sivers effect is sizable for the proton, it becomes negligible for the deuteron, so that apparently the neutron contribution cancels the proton one, showing a strong flavor dependence of the mechanism. Different parameterizations of the available SIDIS data have been published [16, 17, 18], still with large error bars. New data, which will reduce the uncertainties on the extracted Sivers function and will help discriminate between different theoretical predictions, will be available soon.

This experimental scenario motivates theoretical estimates of this quantity. Since a calculation from first principles in QCD is not yet possible, several model evaluations exist, in a quark-diquark model [8, 11, 19]; in the MIT bag model [20]; in a light-cone model [21]; in a nuclear framework, relevant to proton-proton collisions [22]. In here, a Constituent Quark Model (CQM) calculation will be described [23]. CQM calculations of PDs are based on a two steps procedure (see, e.g., Ref. [24]). First, the matrix element of the proper operator is evaluated using the wave functions of the model; then, a low momentum scale is ascribed to the model calculation and QCD evolution is used to evolve the observable calculated in this low energy scale to the high momentum one, where DIS experiments are carried out (see, e.g., Ref [24]). Such procedure has proven successful in describing the gross features of PDs (see, e.g., [25, 26]) and GPDs (see, e.g. [27, 28]), by using different CQMs. Similar expectations motivate the present study of the Sivers function.

The formalism of Ref. [23] to calculate the valence quark contribution to the Sivers function from any CQM is summarized here. A difference in the calculation of TMDs, with respect to calculations of PDs and GPDs, is that the leading twist contribution to the FSI has to be evaluated. All the technical steps of our procedure can be found in Ref. [23], where a workable formula is derived. The main approximations have been: i) only the valence quark sector is investigated; ii) the leading twist FSI are taken into account at leading order, i.e. only one-gluon-exchange (OGE) FSI has been evaluated in the treatment of the gauge link (see Fig. 1); iii) the resulting interaction has been obtained through a non-relativistic (NR) reduction of the relevant operator, according to the philosophy of constituent quark models [29], leading to a potential $V_{N R}$. In terms of the latter, the Sivers function for the quark of flavor $\mathscr{Q}, f_{1 T}^{?} \mathscr{Q}\left(x ; k_{T}\right)$, can be written (cf. 
Fig. 1 for the labels of the momenta and helicities):

$$
f_{1 T}^{? \mathscr{Q}}\left(x ; k_{T}\right)=\mathfrak{I} \quad i g^{2} \frac{M^{2}}{k_{x}} \quad d k_{1} d k_{3} \frac{d^{2} q_{T}}{(2 \pi)^{2}} \delta\left(k_{3}^{+} \quad x P^{+}\right) \delta\left(k_{3 T}+q_{T} \quad k_{T}\right) \mathscr{M}^{\mathscr{Q}}
$$

where $g$ is the strong coupling constant, $M$ the proton mass, and

$$
\begin{aligned}
& \mathscr{M}^{u(d)}=\sum_{m_{1}, m_{1}^{0} m_{3} m_{3}^{0}} \Phi_{s f}^{\dagger} ;_{z}=1 \quad k_{3} ; m_{3} ; k_{1} ; m_{1} ; P \quad k_{3} \quad k_{1} ; m_{n} \\
& \frac{1 \quad \tau_{3}(3)}{2} V_{N R}\left(k_{1} ; k_{3} ; q\right) \\
& \Phi_{s f ;} S_{z}=1 \quad k_{3}+q ; m_{3}^{0} ; k_{1} \quad q ; m_{1}^{0} ; P \quad k_{3} \quad k_{1} ; m_{n} \quad:
\end{aligned}
$$

Eq. (1), with $\mathscr{M}^{u(d)}$ given by Eq. (2), provides us with a suitable formula to evaluate the Sivers function, once the spin-flavor wave function of the proton in momentum space, i.e. the quantity $\Phi_{s f}$, is available in a given constituent quark model. A few remarks are in order. First of all, the helicity conserving part of the global interaction does not contribute to the Sivers function. Besides, one should notice that, in an extreme NR limit, the Sivers function would turn out to be identically zero. In our approach, it is precisely the interference of the small and large components in the four-spinors of the free quark states which leads to a non-vanishing Sivers function, even from the component with $l=0$ of the target wave function. Effectively, these interference terms in the interaction are the ones that, in other approaches, arise due to the wave function (see, e.g., the MIT bag model calculation [20]).

There are many good reasons to use the Isgur-Karl model [30] to test the performance of the approach. First of all, the IK is the typical CQM, successful in reproducing the low-energy properties of the nucleon. In particular, in the IK model, one expects small corrections from terms $O \quad k^{2}=m^{2}$. Besides, one of the features of the IK model is that the OGE mechanism [29], which reduces the degeneracy of the spectrum, is taken into account. It is therefore natural to study our formalism, based on OGE FSI, within the IK framework. Concerning PDs, it has been shown that the IK model can describe their gross features, once QCD evolution of the proper matrix elements of the corresponding twist-2 operators is performed from the scale of the model to the experimental one [24, 25, 26]. Reasonable predictions of GPDs have also been obtained [27], and this makes particularly interesting the evaluation of the Sivers function in the IK model. The final expressions of the Sivers function in the IK model are rather involved and not presented here, since they can be found in Ref. [23].

To evaluate numerically Eq. (11), the strong coupling constant $g$, and therefore $\alpha_{s}\left(Q^{2}\right)$, has to be fixed. Here, the prescription introduced in the past for calculations of PDFs in quark models (see, i.e., Ref. [24]) will be used. It consists in fixing the momentum scale of the model, the so-called hadronic scale $\mu_{0}^{2}$, according to the amount of momentum carried by the valence quarks in the model. In the approach under scrutiny, only valence quarks contribute. Assuming that all the gluons and sea pairs in the proton are produced perturbatively according to NLO evolution equations, in order to have ' $55 \%$ of the momentum carried by the valence quarks at a scale of $0.34 \mathrm{GeV}^{2}$, as in typical lowenergy parameterizations, one finds, that $\mu_{0}^{2}, 0: 1 \mathrm{GeV}^{2}$ if $\Lambda_{Q C D}^{N L O}, 0.24 \mathrm{GeV}$. This 

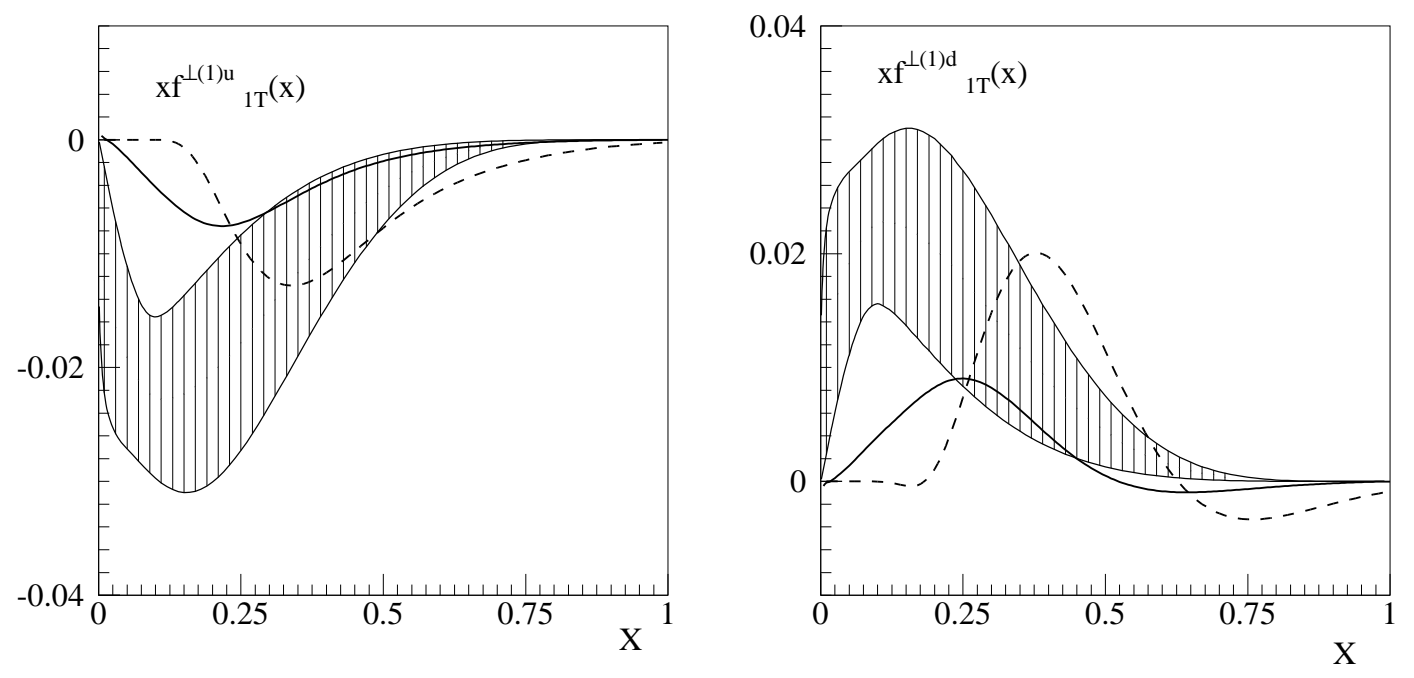

FIGURE 2. Left (right): the quantity $f_{1 T}^{?(1) q}(x)$, Eq. (3), for the $u(d)$ flavor. The dashed curve is the result of the present approach at the hadronic scale $\mu_{0}^{2}$. The full curve represents the evolved distribution after standard NLO evolution (see text). The patterned area represents the $1 \quad \sigma$ range of the best fit of the HERMES data proposed in Ref. [17].

yields $\alpha_{s}\left(\mu_{0}^{2}\right)=(4 \pi)^{\prime} 0: 13[24]$. For an easy presentation, the quantity which is usually shown for the results of calculations or for data of the Sivers function is its first moment, defined as follows :

$$
f_{1 T}^{?(1) \mathscr{Q}}(x)={ }^{2} d^{2} k_{T} \frac{k_{T}^{2}}{2 M^{2}} f_{i T}^{? \mathscr{Q}}\left(x ; k_{T}\right):
$$

The results of the present approach for the moments Eq. (3) are given by the dashed curves in Fig. 2 for the flavors $u$ and $d$. They are compared with a parameterization of the HERMES data, corresponding to an experimental scale of $Q^{2}=25 \mathrm{GeV}^{2}$ [17]. The patterned area represents the $1 \sigma$ range of the best fit proposed in Ref. [17]. Clearly, a different sign for the $u$ and $d$ flavor is found.

Let us see now how the results of the calculation compare with the Burkardt sum rule [31], which follows from general principles and must be satisfied at any scale. If the proton is polarized in the positive $y$ direction, in our case, where only valence quarks are present, the Burkardt sum rule reads:

$$
\sum_{\mathcal{Q}=u x l} \mathrm{k}_{x}^{\mathscr{Q}} \mathrm{i}=0
$$

where

$$
\mathrm{k}_{x}^{\mathscr{Q}} \mathrm{i}={ }_{0}^{\mathrm{Z}_{1}} d x^{\mathrm{z}} d k_{T} \frac{k_{x}^{2}}{M} f_{1 T}^{?} \mathscr{Q}\left(x ; k_{T}\right):
$$

Within our scheme, at the scale of the model, it is found $h k_{x}^{u} \dot{i}=10: 85 \mathrm{MeV}, h k_{x}^{d} \dot{\mathrm{i}}=$ $1125 \mathrm{MeV}$ and, in order to have an estimate of the quality of the agreement of our 
results with the sum rule, we define the ratio

$$
r=\frac{h k_{x}^{d} i+h k_{x}^{u} i}{h k_{x}^{d} i \quad h k_{x}^{u} i} ;
$$

obtaining $r^{\prime} \quad 0 \$ 2$, so that we can say that our calculation fulfills the Burkardt sum rule to a precision of a few percent.

The magnitude of the results is close to that of the data, although they have a different shape: the maximum (minimum) is predicted at larger values of $x$. One should anyway realize that one step of the analysis is still missing: the scale of the model, $\mu_{0}^{2}$, is much lower than the one of the data, which is $Q^{2}=25 \mathrm{GeV}^{2}$. For a proper comparison, the QCD evolution from the model scale to the experimental one would be necessary. Unfortunately, the Sivers function is a TMD PDs and the evolution of this class of functions is, to a large extent, unknown. In order to have an indication of the effect of the evolution, we perform a NLO evolution of the model results assuming, for the moments of the Sivers function, the ones defined in Eq. (3), the same anomalous dimensions of the unpolarized PDFs. As described in the previous section, the parameters of the evolution have been fixed in order to have a fraction ' 055 of the momentum carried by the valence quarks at $0.34 \mathrm{GeV}^{2}$, as in typical parameterizations of PDFs, starting from a scale of $\mu_{0}^{2}, 0: 1 \mathrm{GeV}^{2}$ with only valence quarks. The final result is given by the full curve in Fig. 2 for the $u$ and $d$ flavor. As it is clearly seen, the agreement with data improves dramatically and their trend is reasonably reproduced at least for $x \quad 02$. Of course a word of caution is in order: the performed evolution is not really correct. In any case, an indication of two very important things is obtained: i) The evolution of the model result is necessary to estimate the quantities at the momentum scale of experiments, as it happens for standard PDs [24, 25, 26]; ii) after evolution, the present calculation could be consistent with data, at least with the present ones, still affected by large statistical and systematic errors. One should notice that the agreement which is found is better than that found in other model calculations [19, 20], especially for what concerns the fulfillment of the Burkardt Sum Rule.

\section{THE SIVERS FUNCTION FROM NEUTRON $\left({ }^{3}\right.$ HE) TARGETS}

As we have discussed in the previous section, the experimental scenario which arises from the analysis of SIDIS off transversely polarized proton and deuteron targets [14, 15] is rather puzzling. The data show a strong, unexpected flavor dependence in the azimuthal distribution of the produced pions. With the aim at extracting the neutron information to shed some light on the problem, a measurement of SIDIS off transversely polarized ${ }^{3} \mathrm{He}$ has been addressed [32], and two experiments, planned to measure azimuthal asymmetries in the production of leading $\pi$ from transversely polarized ${ }^{3} \mathrm{He}$, are forth-coming at JLab [33]. Here, a recent, realistic analysis of SIDIS off transversely polarized ${ }^{3} \mathrm{He}$ [34] is described. The formal expressions of the Collins and Sivers contributions to the azimuthal Single Spin Asymmetry (SSA) for the production of leading pions have been derived, in impulse approximation (IA), including also the initial transverse momentum of the struck quark. The final equations are rather involved and they 
are not reported here. They can be found in [34]. The same quantities have been then evaluated in the kinematics of the planned JLab experiments. Wave functions [35] obtained within the AV18 interaction [36] have been used for a realistic description of the nuclear dynamics, using overlap integrals evaluated in Ref. [37], and the nucleon structure has been described by proper parameterizations of data or suitable model calculations [16, 38]. The crucial issue of extracting the neutron information from ${ }^{3} \mathrm{He}$ data will be now thoroughly discussed. As a matter of facts, a model independent procedure, based on the realistic evaluation of the proton and neutron polarizations in ${ }^{3} \mathrm{He}$ [39], called respectively $p_{p}$ and $p_{n}$ in the following, is widely used in inclusive DIS to take into account effectively the momentum and energy distributions of the bound nucleons in ${ }^{3} \mathrm{He}$. It is found that the same extraction technique can be applied also in the kinematics of the proposed experiments, although fragmentation functions, not only parton distributions, are involved, as it can be seen in Figs. 1 and 2. In these figures, the free neutron asymmetry used as a model in the calculation, given by a full line, is compared with two other quantities. One is:

$$
\bar{A}_{n}^{i}, \frac{1}{d_{n}} A_{3}^{\exp i}
$$

where $i$ stands for "Collins" or "Sivers", $A_{3}^{\exp i}$ is the result of the full calculation, simulating data, and $d_{n}$ is the neutron dilution factor. The latter quantity is defined as follows, for a neutron $n$ (proton $p$ ) in ${ }^{3} \mathrm{He}$ :

$$
d_{n(p)}(x ; z)=\frac{\sum_{q} e_{q}^{2} f^{q n(p)}(x) D^{q h}(z)}{\sum_{N=p n} \sum_{q} e_{q}^{2} f^{q N}(x) D^{q h}(z)}
$$

and, depending on the standard parton distributions, $f^{q N}(x)$, and fragmentation functions, $D^{q h}(z)$, is experimentally known (see [34] for details). $\bar{A}_{n}^{i}$ is given by the dotted curve in the figures. The third curve, the dashed one, is given by

$$
A_{n}^{i}, \frac{1}{p_{n} d_{n}} A_{3}^{\text {exp } i} 2 p_{p} d_{p} A_{p}^{\text {exp } i} ;
$$

i.e. ${ }^{3} \mathrm{He}$ is treated as a nucleus where the effects of its complicated spin structure, leading to a depolarization of the bound neutron, together with the ones of Fermi motion and binding, can be taken care of by parameterizing the nucleon effective polarizations, $p_{p}$ and $p_{n}$. One should realize that Eq. (7) is the relation which should hold between the ${ }^{3} \mathrm{He}$ and the neutron SSAs if there were no nuclear effects, i.e. the ${ }^{3} \mathrm{He}$ nucleus were a system of free nucleons in a pure $S$ wave. In fact, Eq. (7) can be obtained from Eq. (9) by imposing $p_{n}=1$ and $p_{p}=0$. It is clear from the figures that the difference between the full and dotted curves, showing the amount of nuclear effects, is sizable, being around $10-15 \%$ for any experimentally relevant $x$ and $z$, while the difference between the dashed and full curves reduces drastically to a few percent, showing that the extraction scheme Eq. (9) takes safely into account the spin structure of ${ }^{3} \mathrm{He}$, together with Fermi motion and binding effects. This important result is due to the peculiar kinematics of the JLab experiments, which helps in two ways. First of all, to favor pions from current 

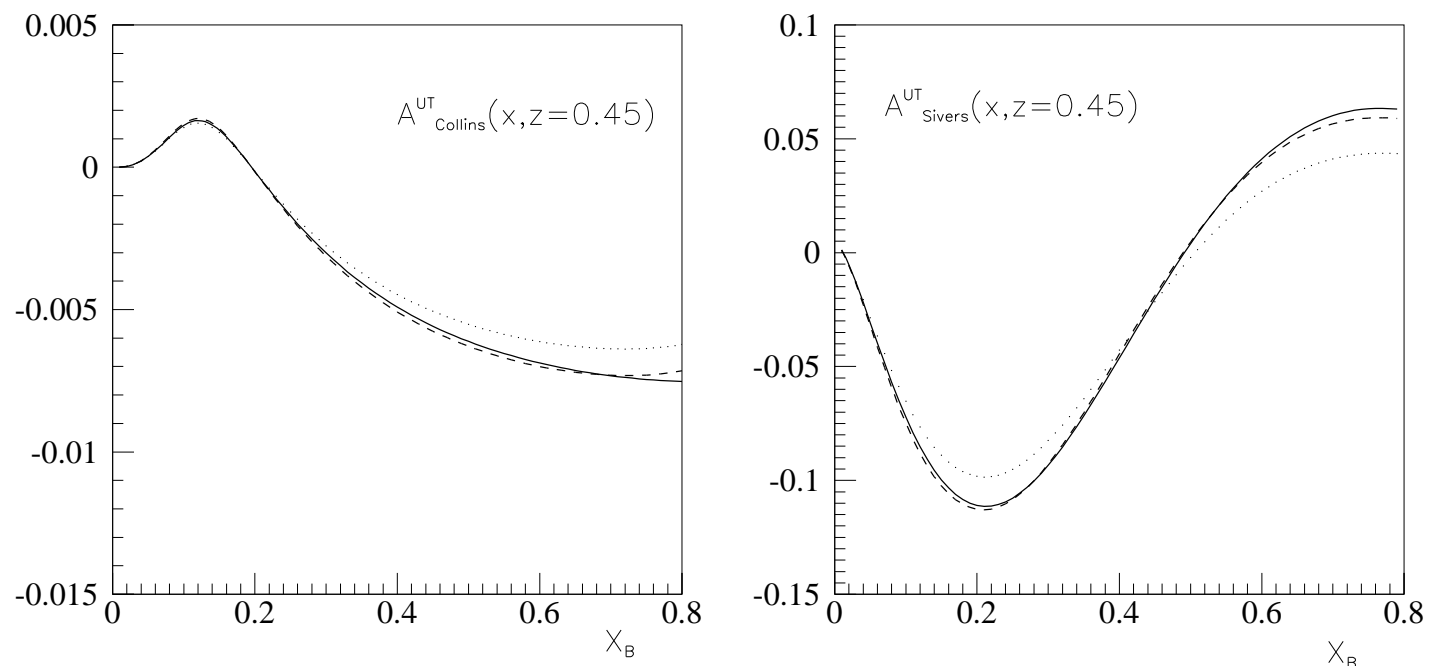

FIGURE 3. Left (right) The model neutron Collins (Sivers) asymmetry for $\pi$ production (full) in JLab kinematics, and the one extracted from the full calculation taking into account the proton effective polarization (dashed), or neglecting it (dotted). The results are shown for $z=0.45$ and $Q^{2}=2.2 \mathrm{GeV}^{2}$, typical values in the kinematics of the JLab experiments.

fragmentation, $z$ has been chosen in the range $0: 45 \quad z \quad 0.6$, which means that only high-energy pions are observed. Secondly, the pions are detected in a narrow cone around the direction of the momentum transfer. As it is explained in [34], this makes nuclear effects in the fragmentation functions rather small. The leading nuclear effects are then the ones affecting the parton distributions, already found in inclusive DIS, and can be taken into account in the usual way, i.e., using Eq. (9) for the extraction of the neutron information. In the figures, one should not take the shape and size of the asymmetries too seriously, being the obtained quantities strongly dependent on the models chosen for the unknown distributions [38]. One should instead consider the difference between the curves, a model independent feature which is the most relevant outcome of the present investigation. The main conclusion is that Eq. (9) will be a valuable tool for the data analysis of the experiments [33].

The evaluation of possible effects beyond IA, such as final state interactions, and the inclusion in the scheme of more realistic models of the nucleon structure, able to predict reasonable figures for the experiments, are in progress.

\section{ACKNOWLEDGMENTS}

It is a pleasure to thank Alessandro Drago and Santiago Noguera for interesting conversations. This work is supported in part by the INFN-CICYT agreement, by the Generalitat Valenciana under the contract AINV06/118; by the Sixth Framework Program of the European Commission under the Contract No. 506078 (I3 Hadron Physics); by the MEC (Spain) under the Contract FPA 2007-65748-C02-01 and the grants AP2005-5331 and PR2007-0048. 


\section{REFERENCES}

1. V. Barone, A. Drago and P. G. Ratcliffe, Phys. Rept. 359 (2002) 1.

2. J. C. Collins, Nucl. Phys. B 396, 161 (1993).

3. P. J. Mulders and R. D. Tangerman, Nucl. Phys. B 461 (1996) 197 [Erratum-ibid. B 484 (1997) 538].

4. A. M. Kotzinian and P. J. Mulders, Phys. Lett. B 406 (1997) 373.

5. D. Boer and P. J. Mulders, Phys. Rev. D 57 (1998) 5780.

6. A. Bacchetta, M. Diehl, K. Goeke, A. Metz, P. J. Mulders and M. Schlegel, JHEP 0702, 093 (2007).

7. D. W. Sivers, Phys. Rev. D 41, 83 (1990), Phys. Rev. D 43, 261 (1991).

8. S. J. Brodsky, D. S. Hwang and I. Schmidt, Phys. Lett. B 530, 99 (2002).

9. S. J. Brodsky, P. Hoyer, N. Marchal, S. Peigne and F. Sannino, Phys. Rev. D 65, 114025 (2002).

10. J. C. Collins, Phys. Lett. B 536, 43 (2002).

11. X. d. Ji and F. Yuan, Phys. Lett. B 543, 66 (2002).

12. A. V. Belitsky, X. Ji and F. Yuan, Nucl. Phys. B 656, 165 (2003).

13. A. Drago, Phys. Rev. D 71, 057501 (2005).

14. A. Airapetian et al. [HERMES Collaboration], Phys. Rev. Lett. 94, 012002 (2005).

15. V. Y. Alexakhin et al. [COMPASS Collaboration], Phys. Rev. Lett. 94, 202002 (2005).

16. M. Anselmino, M. Boglione, U. D’ Alesio, A. Kotzinian, F. Murgia and A. Prokudin, Phys. Rev. D 71, 074006 (2005), M. Anselmino, M. Boglione, U. D’Alesio, A. Kotzinian, F. Murgia and A. Prokudin, Phys. Rev. D 72, 094007 (2005). [Erratum-ibid. D 72, 099903 (2005)]

17. A. V. Efremov, K. Goeke, S. Menzel, A. Metz and P. Schweitzer, Phys. Lett. B 612, 233 (2005); J. C. Collins, A. V. Efremov, K. Goeke, S. Menzel, A. Metz and P. Schweitzer, Phys. Rev. D 73, 014021 (2006).

18. W. Vogelsang and F. Yuan, Phys. Rev. D 72, 054028 (2005).

19. A. Bacchetta, A. Schaefer and J. J. Yang, Phys. Lett. B 578, 109 (2004); A. Bacchetta, F. Conti, M. Radici, arXiv:0807.0323 [hep-ph].

20. F. Yuan, Phys. Lett. B 575, 45 (2003); I. O. Cherednikov, U. D’Alesio, N. I. Kochelev and F. Murgia, Phys. Lett. B 642, 39 (2006).

21. Z. Lu and B. Q. Ma, Nucl. Phys. A 741, 200 (2004).

22. A. Bianconi, arXiv:hep-ph/0702186

23. A. Courtoy, F. Fratini, S. Scopetta, V. Vento, Phys. Rev. D (2008), in press; arXiv:0801.4347[hep-ph].

24. M. Traini, A. Mair, A. Zambarda and V. Vento, Nucl. Phys. A 614, 472 (1997).

25. S. Scopetta and V. Vento, Phys. Lett. B 424, 25 (1998).

26. S. Scopetta and V. Vento, Phys. Lett. B 460, 8 (1999) [Erratum-ibid. B 474, 235 (2000)].

27. S. Scopetta and V. Vento, Eur. Phys. J. A 16, 527 (2003).

28. S. Boffi, B. Pasquini and M. Traini, Nucl. Phys. B 649, 243 (2003).

29. A. De Rujula, H. Georgi and S. L. Glashow, Phys. Rev. D 12, 147 (1975).

30. N. Isgur and G. Karl, Phys. Rev. D 18, 4187 (1978); Phys. Rev. D 19, 2653 (1979). [Erratum-ibid. D 23, 817 (1981)].

31. M. Burkardt, Phys. Rev. D 69 (2004) 091501; Phys. Rev. D 69 (2004) 057501.

32. S.J. Brodsky and S. Gardner, Phys. Lett. B 643, 22 (2006).

33. E-06-010 Proposal to JLab-PAC29, J.-P. Chen and J.-C. Peng Spokespersons; E-06-011 Proposal to JLab-PAC29, E. Cisbani and H. Gao Spokespersons.

34. S. Scopetta, Phys. Rev. D 75, 054005 (2007).

35. A. Kievsky, M. Viviani, and S. Rosati, Nucl. Phys. A 577, 511 (1994).

36. R.B. Wiringa, V.G.J. Stocks, and R. Schiavilla, Phys. Rev. C 51, 38 (1995).

37. A. Kievsky, E. Pace, G. Salmè, and M. Viviani, Phys. Rev. C 56, 64 (1997); E. Pace, G. Salmè, S. Scopetta, and A. Kievsky, Phys. Rev. C 64, 055203 (2001).

38. D. Amrath, A. Bacchetta, and A. Metz, Phys. Rev. D 71, 114018 (2005).

39. C. Ciofi degli Atti, S. Scopetta, E. Pace and G. Salmè, Phys. Rev. C48, 968 (1993). 